\title{
STRAIGHTENING-OUT AND SEMIRIGIDITY IN ASSOCIATIVE ALGEBRAS
}

BY

\author{
FRANCIS J. FLANIGAN( $\left.{ }^{1}\right)$
}

Introduction. Let $A=N \oplus S$ be a finite-dimensional associative algebra over the perfect field $k$, here written as the semidirect sum of its radical $N$ and a separable Wedderburn factor $S$. We do not require that $A$ have a multiplicative unit. Denote the multiplication in $A$ by $\pi(x, y)=x y$. Suppose we are given a deformation of the algebra structure on $A$ via the associative bilinear map $\pi_{t}(x, y)=x y+t F_{1}(x, y)$ $+t^{2} F_{2}(x, y)+\cdots$; here $\pi_{t}$ is the multiplication in the generic element $A_{t}$ of a one-parameter family of deformations of $A$ in the sense of Gerstenhaber [3, p. 62]. (Recall that $A_{t}$ is an algebra over the scalar field $K=k((t))$, the field of power series over $k$ in an indeterminate $t$.) It is natural to ask how the structure of the deformed algebra $A_{t}$ compares with that of $A$ (or, to be technically precise, with that of $A_{K}$, the algebra with multiplication $\pi$ but scalars extended to $K$ ). For example, if $A=S$ is separable semisimple, then $A$ is rigid and $A_{t}$ is isomorphic to $A_{K}$. At the opposite extreme, however, if $A=N$ is nilpotent, then it is highly deformable (see a forthcoming paper), and $A_{t}$ may be quite unlike $A_{K}$.

In the first part of this paper we state a result (Theorem 1) which straightensout (as far as possible) the deformation $\pi_{t}$. That is, we construct a deformation $\mu_{t}$ equivalent to $\pi_{t}$ (see [3, p. 65]) but better behaved as follows: $\mu_{t}$ does not subject the underlying subspaces of the radical $N$ and Wedderburn factor $S$ to a meaningless rotation, and $\mu_{t}$ respects the rigidity of the semisimple $S$ by not introducing trivial deformations here. This enables us to compare $A_{t}$ (multiplication $\mu_{t}$ now) with $A_{K}$. The view thus afforded leads us to define semirigidity as a natural generalization of rigidity; see $\$ 2$. Briefly, semirigid algebras are those which cannot become more semisimple through deformation. In $\S 3$ and $\S 4$ we examine in some detail the two types of deformation which increase semisimplicity. Our analysis yields explicit deformation formulae and a method of constructing semirigid algebras.

An earlier version of Theorem 1 appeared in the author's thesis, written with the guidance of Professor Maxwell Rosenlicht at Berkeley. The author is grateful for this assistance, and is pleased also to acknowledge many discussions of deformation theory with Drs. Murray Gerstenhaber, Alan Landman and Albert Nijenhuis.

1. The straightening-out theorem. Roughly put, this will tell us (i) the radical $N_{K}$ of $A_{K}$ shrinks under deformation to that of $A_{t}$, while a Wedderburn factor

Received by the editors August 8, 1967 and, in revised form, May 28, 1968.

( $\left.{ }^{1}\right)$ The final stages of this research were supported by NSF GP 6895. 
of $A_{t}$ contains $S_{K}$ and absorbs part of $N_{K}$, (ii) the multiplication in the subalgebra $S_{K}$ is unchanged, (iii) the two-sided regular action of $S_{K}$ on $N_{K}$ is unchanged.

In what follows we work in the context of the Wedderburn Principal Theorem; that is, all algebras have a semidirect sum decomposition (radical) $\oplus$ (semisimple); this is always the case in characteristic zero. Also, $V$ will denote the underlying $k$-space of $A$; we write $A=\operatorname{alg}(V, \pi)$ and similarly for other algebras. For any $k$-space $U$ we denote the scalar extension $U \otimes_{k} K$ by $U_{K}$. It will often be convenient to suppose $U$ is imbedded in $U_{K}$. All other definitions and notations are in [3]. The above description can now be made precise.

THEOREM 1. Let $A=N \oplus S$ and $\pi, \pi_{t}$ be as above. Then

(a) there exists a $K$-space decomposition $N_{K}=M_{K} \oplus T$, where $M$ (resp. $T$ ) is a $k$ - (resp. $K$-) subspace of $N$ (resp. $N_{K}$ ), and

(b) there exists a generic deformation $\mu_{t}(x, y)=x y+t G_{1}(x, y)+\cdots$ of $A$ equivalent to $\pi_{t}$ such that

(c) $M=\operatorname{alg}(M, \pi)$ is a nilpotent ideal of $A$,

(d) $M_{t}=\operatorname{alg}\left(M_{K}, \mu_{t}\right)$ is the radical of the $K$-algebra $A_{t}=\operatorname{alg}\left(V_{K}, \mu_{t}\right)$,

(e) $\mu_{t}(x, y)=x y$ for all $x, y \in S_{K}$,

(f) $\mu_{t}(x, z)=x z$ and $\mu_{t}(z, x)=z x$ for $x \in S_{K}, z \in N_{K}$,

(g) $T$ is an $S_{K}$-bimodule in both $A_{K}$ and $A_{t}$,

(h) $W_{t}=\operatorname{alg}\left(T \oplus S_{K}, \mu_{t}\right)$ is a semisimple Wedderburn factor of $A_{t}$, so that $A_{t}=M_{\iota} \oplus W_{t}$.

Proof. This is lengthy but elementary; we omit many details. It is important to construct intermediate equivalences in the proper order. (i) First we deal with the radicals, proving (c), (d) and part of (a). Let $\zeta_{1}, \ldots, \zeta_{r}$ be a $K$-basis for $\operatorname{rad}\left(\pi_{t}\right)$, a subspace of $V_{K}$. Without loss $\zeta_{i}=z_{i}+$ (powers of $t$ with coefficients in $V$ ) and the $z_{i}$ are $k$-independent. Using the definition of [7, p. 140], one shows that the $z_{i}$ are in the radical $N$ of $A$. Define $M$ to be the $k$-space with basis $z_{1}, \ldots, z_{r} . M$ is an ideal for the multiplication $\pi$ because $\operatorname{rad}\left(\pi_{t}\right)$ is for $\pi_{t}$; this gives (c) above. Now extend $z_{1}, \ldots, z_{r}$ to a $k$-basis of $V$ which is thereby a $K$-basis of $V_{K}$. Define $\Phi_{t}\left(\zeta_{i}\right)=z_{i}$ and extend to all of $V_{K}$ using the $k$-basis so that $\Phi_{t}$ has the form id $+t \phi_{1}+\cdots$. The multiplication $\Phi_{t} \circ \pi_{t} \circ\left(\Phi_{t}^{-1} \times \Phi_{t}^{-1}\right)$ is equivalent to $\pi_{t}$ and satisfies (d) above.

(ii) Now suppose $\pi_{t}$ has the properties established in (i). We obtain (e) and (f) by applying a miniature deformation theory of algebra homomorphisms which we now sketch. Let $A, B$ be $k$-algebras, $B$ with unit 1 , and let $f: A \rightarrow B$ be an algebra homomorphism which need not map unit to unit. Thus $B$ is an $(A, f)$-bimodule. A deformation of $f$ is given by a $K$-algebra homomorphism $f_{t}: A_{K} \rightarrow B_{K}$ of the form $f_{t}=f+t F_{1}+\cdots$, where $F_{i}: A \rightarrow B$ is $k$-linear. We say $f_{t}$ is trivial iff there is an inner automorphism $\beta_{t}: B_{K} \rightarrow B_{K}$ given by conjugating with an element $1+t b_{1}+\cdots$ of $B_{K}$ such that $f_{t}=\beta_{t} \circ f$. We say $f$ is rigid iff all such deformations $f_{t}$ are trivial. Now let $H^{*}(A, f, B)$ denote Hochschild cohomology of $A$ with coefficients in the $(A, f)$-bimodule $B$. One proves, in analogy with $[3, \mathrm{p} .65]$, the 
Lemma (Nijenhuis). If $H^{1}(A, f, B)=(0)$, then $f$ is rigid.

To obtain (e) and (f), form $\Sigma=S \otimes_{k} S^{\text {op }}$ and map $f: \Sigma \rightarrow$ End ( $V$ ) by $\left(f\left(x \otimes x^{\prime}\right)\right) y=x y x^{\prime}$ for all $x, x^{\prime} \in S$ and $y \in A$. Now write $\pi_{t}\left(y, y^{\prime}\right)=y * y^{\prime}$ for all $y, y^{\prime} \in V_{K}$. Then $f_{t}: \Sigma_{K} \rightarrow$ End $(V)_{K}$ defined by $\left(f_{t}\left(x \otimes x^{\prime}\right)\right) y=x * y * x^{\prime}$ is a deformation of $f$. Since $\Sigma$ is separable semisimple, $f_{t}$ must be trivial. Thus there is a $K$-linear automorphism $\Xi_{t}$ of $V_{K}$ of the form $\Xi_{t}(x)=x+t \xi_{1}(x)+\cdots$ such that the composition $\Xi_{t}^{-1} \circ \pi_{t} \circ\left(\Xi_{t} \times \Xi_{t}\right)$ satisfies (e) and (f) as well as (d). We call this multiplication $\mu_{t}$.

(iii) Now we obtain $W_{t}, T$, (a), (g) and (h). Let $W_{t}$ be a Wedderburn factor containing the subalgebra $S_{K}$ of $A_{t}=\operatorname{alg}\left(V_{K}, \mu_{t}\right)$. Define the $K$-subspace $T$ as $N_{K} \cap W_{t}$. By dimension, (a) holds. Also, by (f), $\mu_{t}\left(S_{K}, T\right)=\pi\left(S_{K}, T\right) \subset N_{K}$, and since $S_{K}$ and $T$ are contained in $W_{t}$ we have $\mu_{t}\left(S_{K}, T\right) \subset W_{t}$. Thus $\mu_{t}\left(S_{K}, T\right) \subset T$ and $(\mathrm{g})$ follows. The definition of $T$ yields (h), and the theorem is proved.

A corollary (not new) of (e) is: The dimension of the radical does not increase under deformation.

The following illustrates the theorem, underlining the facts that the radical decomposes over $K$ but not, in general, over $k$ and also that $T$ need not be a subalgebra of $A_{K}$. Let $A=N$ have basis $x, y, z$ over $k$ and multiplication $x y=z$, all other products of basis elements zero. Now obtain $A_{t}$ over $K$ by setting $\pi_{t}(x, y)$ $=x y=z, \pi_{t}(x, x)=t x, \pi_{t}(y, y)=t y, \pi_{t}(x, z)=\pi_{t}(z, y)=t z$, other products zero. Let $E$ denote the (rigid) algebra of two-by-two matrices over $K$ with lower left-hand corners zero and $e_{11}, e_{12}, e_{22}$ the usual basis. Then the map $t^{-1} x \rightarrow e_{11}, t^{-1} y \rightarrow e_{22}$, $t^{-2} z \rightarrow e_{12}$ is a $K$-algebra isomorphism $A_{t} \rightarrow E$. In the language of the theorem, $A_{t}=M_{t} \oplus T$, where $z$ is a $k$-basis for $M$ and $t^{-1} x-t^{-2} z, t^{-1} y$ is a $K$-basis of orthogonal idempotents for $T=W_{t}$. And $T$ clearly has no $k$-basis. For the nilpotent deformations of $A$, see [3, p. 91].

An analogous theorem for Lie algebras has been obtained by Page and Richardson [6], using the geometric methods of [5]. The proof of parts (e) and (f) of Theorem 1 above shows, in fact, that if $S$ is any subalgebra of $A$, not necessarily semisimple, such that the group $H^{1}\left(S \otimes_{k} S^{\text {op }}\right.$, End $\left.(V)\right)$ is zero, then $S$ is "stable" under deformations of $A$, as is the regular action of $S$ on the bimodule $A$.

We shall use Theorem 1 repeatedly in $\S 3$ and $\S 4$ to examine the types of deformation introduced there. However, let us exercise it immediately (i) to construct some rigid algebras, and (ii) to prove the algebra of all upper-triangular matrices rigid.

(i) An example reveals the method of construction. Let $S_{1}, S_{2}$ be any separable $k$-algebras with units $e_{1}, e_{2}$. Let $N$ be any $\left(S_{1}, S_{2}\right)$-bimodule. Form the associative algebra $A=N \oplus S$, with $S$ the ideal direct sum $S_{1} \oplus S_{2}$, by defining $N^{2}=N e_{1}$ $=e_{2} N=(0)$. Then $A$ is rigid: for let $\mu_{t}(x, y)=x * y$ determine a straightened-out deformation of $A$. By Theorem 1 only products of elements $z, z^{\prime} \in N$ may deform. But $z * z^{\prime}=\left(z e_{2}\right) *\left(e_{1} z^{\prime}\right)=z\left(e_{2} e_{1}\right) z^{\prime}=0$, whence $\mu_{t}$ is trivial as desired. The idea here, of course, is that the multiplication in $N$ is constrained by the rigid action of $S$ 
to be zero both before and after deformation. This observation may be stated as a theorem. We have $A=N \oplus S$ with $S=S_{1} \oplus \cdots \oplus S_{s}$ the direct sum of separable simple $S_{\alpha}$ with unit $e_{\alpha}$. Let $N_{\alpha \beta}=e_{\alpha} N e_{\beta}$. Form $e=e_{1}+\cdots+e_{s}$ which is idempotent but need not be a two-sided identity. Now define

$$
N_{0 \alpha}=\left\{z \in N \mid e z=0 \text { and } z e_{\alpha}=z\right\}
$$

and likewise for $N_{\alpha 0}$ and $N_{00}$. Arguing as above we have

THEOREM 1.1. Let $A=N \oplus S$ over the perfect field $k$. Then $N^{2}=(0)$ and $A$ is rigid iff

(a) $N_{\alpha \alpha}=(0)$,

(b) if $N_{\alpha \beta}>(0)$, then $N_{\beta \alpha}=(0)$, and

(c) if $N_{\alpha \beta}>(0)$ and $N_{\beta \gamma}>(0)$, then $N_{\alpha \gamma}=(0)$ for $\alpha, \beta, \gamma=0,1, \ldots, s$.

(ii) Now we consider an algebra with $N^{2}>(0)$. Let $\nabla=\nabla(r, k)$ be the algebra of all $r$ by $r$ matrices over $k$ which have only zero entries below the main diagonal. $\nabla$ has the usual $k$-basis $e_{\alpha \beta}$, with $1 \leqq \alpha \leqq \beta \leqq r$, satisfying $e_{\alpha \beta} e_{\beta \gamma}=e_{\alpha \gamma}$ while $e_{\alpha \beta} e_{\gamma \delta}=0$ for $\beta \neq \gamma$.

The following seems to be widely believed. We offer a constructive proof.

THEOREM 1.2. $\nabla(r, k)$ is rigid.

Proof. Let a deformation $\mu_{t}$ be straightened-out as in Theorem 1. Then products involving the $e_{\alpha \alpha}$ are unchanged, while by part (f) we conclude that $e_{\alpha \beta} * e_{\beta \gamma}$ $=\mu_{t}\left(e_{\alpha \beta}, e_{\beta \gamma}\right)=\xi_{\alpha \beta \gamma} e_{\alpha \gamma}$, with $\xi_{\alpha \beta \gamma} \in k[[t]]$ and constant term $\xi_{\alpha \beta \gamma}(0)=1$. To prove $\mu_{t}$ trivial, one notes that it suffices to prove the existence of $\eta_{\alpha \beta} \in k[[t]]$ with constant term $\eta_{\alpha \beta}(0)=1$ such that the "perturbed" basis $e_{\alpha \beta}^{\prime}=\eta_{\alpha \beta} e_{\alpha \beta}$ satisfies $e_{\alpha \beta}^{\prime} * e_{\beta \gamma}^{\prime}=e_{\alpha \gamma}^{\prime}$. Clearly, this is possible when $r=1,2,3$. We suppose the theorem is true in rank $r-1$ and proceed by induction. Thus the subalgebra $B$ of $\nabla$ with basis $e_{\alpha \beta}$, $1 \leqq \alpha \leqq \beta \leqq r-1$, is rigid and the restriction of $\mu_{t}$ to $B$ is trivial. Hence there exist $\eta_{\alpha \beta}$ and $e_{\alpha \beta}^{\prime}$ as required, except when $\beta=r$; now we treat the right-hand column. First put $\eta_{1 r}=1$, so that $e_{1 r}^{\prime}=e_{1 r}$. Now define $\eta_{2 r}$ so that $e_{12}^{\prime} * e_{2 r}^{\prime}=e_{1 r}^{\prime}$, where $e_{2 r}^{\prime}=\eta_{2 r} e_{2 r}$. This is always possible. (Note that only $e_{12}^{\prime}$ multiplies $e_{2 r}$ on the left, except for the left identity $e_{22}^{\prime}=e_{22}$.)

Now, in the third row, define $\eta_{3 r}$ so that $e_{23}^{\prime} * e_{3 r}^{\prime}=e_{2 r}^{\prime}$ defined previously. Then we note that $e_{13}^{\prime} * e_{3 r}^{\prime}=e_{12}^{\prime} *\left(e_{23}^{\prime} * e_{3 r}^{\prime}\right)=e_{1 r}^{\prime}$, the desired product. Continue down the column, always defining $\eta_{p r}$ so that $e_{q p}^{\prime} * e_{p r}^{\prime}=e_{q r}^{\prime}$ where $q=p-1$. The theorem follows.

The elementary reduction of $\S 4$, (ii) immediately yields the rigidity of the "block upper-triangular" matrix algebra obtained from $\nabla$ by replacing each $e_{\alpha \alpha}$ by an algebra $S_{\alpha}$ of all $r_{\alpha}$ by $r_{\alpha}$ matrices over $k$ and each $e_{\alpha \beta}$ with $\alpha<\beta$ by an $r_{\alpha} r_{\beta}$-dimensional $\left(S_{\alpha}, S_{\beta}\right)$-bimodule.

(ADDENDum. Both David Knudson and the referee have pointed out that $\nabla$ has global dimension one, whence $H^{2}(\nabla, \nabla)=0$ and Theorem 1.2. Cf. Eilenberg, Rosenberg and Zelinsky in Nagoya Math J. 12 (1957), or M. Auslander's Brandeis notes “ Rings, modules and homology.") 
2. Semirigidity. We noted above that deformation cannot increase the dimension of the radical. Let us generalize the notion of rigidity as follows: we say that $A$ is semirigid over $k$ iff $A$ admits no deformation over $k$ which decreases the dimension of the radical. Thus, in the language of Theorem $1, A$ is semirigid iff for all deformations $\mu_{t}$ the corresponding subspace $T$ (which measures the growth in semisimplicity) is zero. Semirigid algebras are natural objects of study for the following reasons: (a) every deformation thereof is simply the deformation of a nilpotent multiplication, and (b) every associative algebra can be deformed into a semirigid algebra,-but not necessarily into a rigid algebra. (See a forthcoming paper [2].) As we are about to see, moreover, certain tests for semirigidity involve only rather coarse structural invariants of $A$ (e.g. dimension of certain subspaces, solvability of some finite linear relations) which often can be readily checked for a given multiplication table.

Finally, one may phrase the problem of finding all rigid algebras as follows: select from among the semirigid algebras those algebras $A=N \oplus S$ whose radicals $N$, constrained by the two-sided action of $S$ (rigid by Theorem 1), admit no deformations. The basic Rigidity Theorem has its analogue here.

To facilitate the discussion, from now on $A=N \oplus S$ will have unit $e$ and the scalar field $k$ will be perfect. Also $S=S_{1} \oplus \cdots \oplus S_{s}$ is an ideal direct sum of simple algebras, with each $S_{\alpha}$ by fiat a total $r_{\alpha}$ by $r_{\alpha}$ matrix algebra over $k$ with unit $e_{\alpha}$; we do not treat division algebras over $k$. Note $e=e_{1}+\cdots+e_{s}$. Writing $N_{\alpha \beta}$ for $e_{\alpha} N e_{\beta}$, we have $N=\oplus N_{\alpha \beta}$ with $\alpha, \beta=1, \ldots, s$; this is a so-called Peirce decomposition of the radical into a direct sum of subalgebras. Note $N_{\alpha \beta} N_{\gamma \delta}=(0)$ if $\beta \neq \gamma$.

Now we observe, using Theorem 1 , that a deformation with $T \neq(0)$ may combine the following two phenomena:

(I) A subalgebra of the radical $N_{K}$ may deform into a total matrix algebra. In particular, a radical element becomes, in the new multiplication, an idempotent. Example: let $A$ have basis $z, e$ with $z^{2}=0, e^{2}=e=$ the two-sided identity (unit) of $A$. Deform via $\mu_{t}(z, z)=t z$, other products among basis elements as before. Then the $K$-algebra $A_{t}$ is isomorphic to the ideal direct sum $K \oplus K$, and the element $u=t^{-1} z$ is an idempotent for $\mu_{t}$.

(II) Under deformation, a subspace of the radical $N_{K}$ (in fact, of $T$ ) may coalesce with two or more matrix subalgebras of $S_{K}$ to form one larger matrix algebra. In this case, no idempotents are produced from radical elements alone; all idempotents of $A_{t}$ "involve" idempotents of $S_{K}$.

Example: Let $A$ be four-dimensional, with $z_{12}, z_{21}$ a basis for $N$, satisfying $N^{2}=(0)$, and $e_{1}, e_{2}$ orthogonal idempotents in $S$. For distinct $\alpha, \beta=1,2$ let $e_{\alpha} z_{\alpha \beta}$ $=z_{\alpha \beta} e_{\beta}=z_{\alpha \beta}$ (this is essential) with other products zero. Now obtain $A_{t}$ via the deformation $\mu_{t}\left(z_{\alpha \beta}, z_{\beta \alpha}\right)=t^{2} e_{\alpha}$, with other products as before. We see that the map $t^{-1} z_{\alpha \beta} \rightarrow e_{\alpha \beta}, e_{\alpha} \rightarrow e_{\alpha \alpha}$ gives a $K$-isomorphism $A_{t} \rightarrow M(2, K)=$ the algebra of 2 by 2 matrices over $K$ with usual basis $e_{\alpha \beta}$.

Following this analysis we make two more definitions. Let $\pi_{t}$ determine a de- 
formation of $A$ and suppose, further, that $\pi_{t}$ is straightened-out as in the conclusion of Theorem 1. Thus $A_{t}=\operatorname{alg}\left(V_{K}, \pi_{t}\right)=M_{t} \oplus T \oplus S_{K}$. We say that $\pi_{t}$ gives a deformation of Type I iff some element of $T$ is an idempotent for $\pi_{t}$. We say that $\pi_{t}$ gives a deformation of Type II iff $T \neq(0)$ but (as in example (II)) no element of $T$ is an idempotent for $\pi_{t}$. Thus $A$ is not semirigid iff it admits a deformation of either type. We shall study semirigidity from this point of view in the next two sections.

3. Deformations of Type I. First we present a readily applicable necessary condition for Type I deformation. The argument is clarified by writing the deformed product $\pi_{t}(x, y)$ as $x * y$. It is essential to note that if $\pi_{t}$ is straightened-out, then $N_{\alpha \beta} * N_{\gamma \delta}=(0)$ for $\beta \neq \gamma$.

THEOREM 2. If $A$ admits a Type I deformation, then some $N_{\alpha \alpha}$ is nonzero.

Proof. Let $u \in N_{K}$ satisfy $u * u=u$. We have $u=\sum u_{\gamma \delta}$ with $\gamma, \delta=1, \ldots, s$ where $u_{\gamma \delta}=e_{\gamma} u e_{\delta}=e_{\gamma} * u * e_{\delta}$. Let $u_{\gamma \delta}$ be a nonzero term in this sum. Repeated multiplications of $u$ by itself allow us to write $u_{\gamma \delta}$ as a sum of terms of the form $u_{\gamma \varepsilon} * u_{\varepsilon \zeta} * \cdots * u_{\omega \delta}$. After a finite number of such multiplications, each such term must involve a repeated subscript; that is, each term has a factor of the form $u_{\alpha \beta} * \cdots * u_{\theta \alpha}$ and not all of these are zero. Thus some $\left(N_{\alpha \alpha}\right)_{K}$ contains a nonzero element, and the theorem follows immediately.

Thus the algebra of Example (II) in the previous section could not have admitted a Type I deformation.

We have good reason to believe that the condition of Theorem 2 is not sufficient for Type I deformability, but the evidence would take us too far afield now. See [2]. The search for sufficient conditions partakes, of course, of the general question of existence of deformations, which is far from solution. Thus, it is known that if the Hochschild group $H^{3}(A, A)=(0)$, then every 2-cocycle of $A$ may be "integrated" to give a (possibly trivial) deformation. And Gerstenhaber has shown that the cup product of commuting derivations of $A$ may be exponentiated in characteristic zero to a deformation. (See [3, p. 64] and [4].) Neither of these results, however, gives explicit information about the production of idempotents. It is possible to concoct various ad hoc statements. The following lemma is an example. The proof is straightforward.

Lemma 3. Let $z \in N_{\alpha \alpha} \cap \operatorname{Ann}(N)$ and $z \notin N^{2}$. Then there is a deformation of $A$ for which $t^{-1} z$ is an idempotent.

4. Deformations of Type II. Suppose $A$ admits a Type II deformation $\pi_{t}$. What must $A$ look like? What of $\pi_{t}$ ? Is it equivalent to a deformation of particularly simple form? And what conditions on $A$ guarantee Type II deformability? Theorem 4 below provides an answer to the first question, describing the "size and shape" of the radical $N$ as an $S$-bimodule. In Theorem 5 we see that the "pre-matrix" part of $\pi_{t}$ can essentially be given by polynomials in $t$; such theorems (see also [1]) assure us that the explicit deformations we construct by trial and 
error, adjusting various powers of $t$, are, in the sense of equivalence, all. And in Theorem 6 we give sufficient conditions for Type II deformability, reducing the problem to that of finding a nilpotent deformation within the radical. For low dimensions this is often practicable. In this regard, the principle enunciated in (ii) below may be useful.

(i) As above $k$ is perfect, $A=N \oplus S$ with unit $e, S=S_{1} \oplus \cdots \oplus S_{\mathrm{s}}$ is a direct sum of $k$-matrix algebras, so that $e=e_{1}+\cdots+e_{s}$, and $N=\oplus N_{\alpha \beta}$ where $N_{\alpha \beta}$ $=e_{\alpha} N e_{\beta}$. We note now that $N_{\alpha \beta}$ is (nonuniquely) the module direct sum of simple $S_{\alpha}, S_{\beta}$-bimodules $N_{\alpha \beta i}$ (so-called $\alpha \beta$-blocks) of dimension $r_{\alpha} r_{\beta}$ (recall $r_{\alpha}=$ rank of $S_{\alpha}$ ).

Now let $e_{\alpha \theta \lambda}$ with $\theta, \lambda=1, \ldots, r_{\alpha}$ be the usual matrix basis for $S_{\alpha}$. Then each $\alpha \beta$-block $N_{\alpha \beta i}$ has a basis $z_{\alpha \beta i \mu \nu}$ with $\mu=1, \ldots, r_{\alpha}$ and $\nu=1, \ldots, r_{\beta}$ which satisfies $e_{\alpha \theta \lambda} z_{\alpha \beta i \mu \nu} e_{\beta \rho \tau}=\delta_{\lambda \mu} \delta_{\nu \rho} z_{\alpha \beta i \theta \tau}$ (Kronecker deltas). With such a basis for $N$ we observe that if, say, $z_{\alpha \beta h 11} z_{\beta \gamma i 11}=\sum_{j} c_{h i j} z_{\alpha \gamma j 11}$, then also $z_{\alpha \beta h \theta \lambda} z_{\beta \gamma i \lambda \mu}=\sum_{j} c_{h i j} z_{\alpha \gamma j \theta \mu}$ for all $\theta, \lambda, \mu$. That is, the product $z_{\alpha \beta h 11} z_{\beta \gamma i 11}$ determines $N_{\alpha \beta h} N_{\beta \gamma i}$.

(ii) These considerations lead us to construct the subalgebra (different unit) $A^{\prime}=N^{\prime} \oplus S^{\prime}$ of $A$ as follows: let $S^{\prime}$ have $k$-basis $e_{\alpha 11}$ (now written as $e_{\alpha}$ ) with $\alpha=1, \ldots, s$ as usual, and let $N^{\prime}$ have $k$-basis $z_{\alpha \beta i 11}$ (now written as $z_{\alpha \beta i}$ ) with $\alpha, \beta=1, \ldots, s$ and one index $i$ for each $N_{\alpha \beta i}$. We multiply in $A^{\prime}$ as in $A$. The following folk theorem will be useful.

Reduction Principle. The subalgebra $A^{\prime}$ entirely captures the structure of $A$. Given the ranks $r_{1}, \ldots, r_{s}, A$ can be recovered from $A^{\prime}$. Moreover, a straightened-out deformation $\pi_{t}$ of $A^{\prime}$. can be defined immediately on all of $A$.

Thus the number $s$ of simple factors $S_{\alpha}$, along with the numbers of the various $\alpha \beta$-blocks $N_{\alpha \beta i}$, provide a better index than does $\operatorname{dim} A$ to the amount of computation involved in deforming $A$.

(iii) Now suppose $\pi_{t}$ determines a straightened-out Type II deformation of $A$. Thus $A_{t}=\operatorname{alg}\left(V_{K}, \pi_{t}\right)=M_{t} \oplus T \oplus S_{K}$, where the subalgebra $T \oplus S_{K}$ is the Wedderburn factor $W_{t}$ of $A_{t}$. Suppose further that $W_{t}=\Sigma_{1} \oplus \Sigma_{2} \oplus \cdots \oplus \Sigma_{\sigma}$ is a direct sum of matrix algebras with coefficients in $K$; we rule out discussion of division algebras over $K$. Now there is no loss in supposing that the index set $\{1, \ldots, s\}$ partitions into disjoint sets $\langle 1\rangle,\langle 2\rangle, \ldots,\langle\sigma\rangle$ with $\sigma<s$ such that the simple algebra $\Sigma_{p}$ contains the semisimple $S_{\langle p\rangle}=\oplus S_{\alpha}, \alpha \in\langle p\rangle, p=1, \ldots, \sigma$ and, moreover, that rank $\Sigma_{p}=r_{\langle p\rangle}=$ the sum of those ranks $r_{\alpha}$ with $\alpha \in\langle p\rangle$. Here, of course, we are identifying the underlying $k$-space $V$ of $A$ with a subset of $V_{K}$, via the basis of (i) above, say. We note $e_{\langle p\rangle}=$ the unit of $\Sigma_{p}=$ the sum of those $e_{\alpha}$ with $\alpha \in\langle p\rangle$. And, further, the diagonal blocks of the matrices in $\Sigma_{p}$ are filled by the elements of $\left(S_{\langle p\rangle}\right)_{K}$.

(iv) Now let the set $\langle p\rangle$ contain more than one element from $\{1, \ldots, s\}$. The matrix algebra $\Sigma_{p}$ thereby has off-diagonal blocks which are filled by elements of $T$; these latter were radical elements for the original multiplication $\pi$. Cf. $z_{12}, z_{21}$ in Example (II) of $\$ 2$. In accord with that example, it is entirely straightforward to 
show: for each ordered pair of distinct $\alpha, \beta \in\langle p\rangle$, there exists an $\alpha \beta$-block $N_{\alpha \beta 0}$ such that, writing $U_{p}=\oplus N_{\alpha \beta 0}$ with distinct $\alpha, \beta \in\langle p\rangle$, the off-diagonal blocks of $\Sigma_{p}$ are filled by $\left(U_{p}\right)_{K}$. Also $U_{p}$ is an $S_{\langle p\rangle}$-bimodule and the $k$-algebra $P_{p}=U_{p} \oplus S_{\langle p\rangle}$ deforms via $\pi_{t}$ into $\Sigma_{p}$. Thus $P_{p}$ is a pre-matrix algebra in the language of [1], that is, a $k$-algebra with unit which deforms into the algebra of all $K$-matrices of a given rank, while maintaining the same unit.

And, finally, we form the $k$-algebra $U=\oplus U_{p}, p=1, \ldots, \sigma$; this is a direct sum of nilpotent ideals. Then we note that $T=U_{K}$, -in this case the $K$-subspace $T$ of Theorem 1 does have a $k$-basis and, moreover, is a subalgebra in the original multiplication.

(v) Now we shall see what the two-sided action of $W_{t}$ on the radical $M_{t}$ implies about $A$. As in Theorem 1 , the underlying $K$-space of $M_{t}$ is $M_{K}$, where $M$ is a $k$-subspace of the radical $N$ and, in fact, an ideal of $A$. It follows that $M=\oplus M_{p q}$ with $p, q=1, \ldots, \sigma$, where $M_{p q}=e_{\langle p\rangle} M e_{\langle q ;}$ as usual. Further, each $M_{p q}$ is a direct sum of $p q$-blocks $M_{p q i}$. And since $e_{\langle p ;}$ is the sum of those $e_{\alpha}$ with $\alpha \in\langle p\rangle$, we may say that $M_{p q i}=\oplus N_{\alpha \beta i}$, where $i \geqq 1$ is fixed and the sum is taken over all $\alpha \in\langle p\rangle$, $\beta \in\langle q\rangle$.

Let us define $n_{p q}$ by the formula: $K$-dim $M_{p q}=n_{p q} r_{\langle p\rangle} r_{\langle q\rangle}$. Thus $n_{p q}$ is the number of $p q$-blocks in a decomposition of $M_{p q}$. Now we do some counting. Since each $M_{p q i}$ is composed of all $N_{\alpha \beta i}$ with $i \geqq 1$ and $\alpha \in\langle p\rangle, \beta \in\langle q\rangle$, the number of $N_{\alpha \beta i}$ with $i \geqq 1$ must depend only on the index $p q$ and is, in fact, $n_{p q}$. These considerations, plus recollection of the blocks $N_{\alpha \beta 0}$ in (iv) above, yield the conclusions about $\operatorname{dim} N_{\alpha \beta}$ stated in (c) below. We sum up our observations of the last three paragraphs in the following theorem.

THEOREM 4. Let $A=N \oplus S$ be a finite-dimensional algebra with unit over the perfect field $k$, with $S=S_{1} \oplus \cdots \oplus S_{s}$ a direct sum of total $k$-matrix algebras $S_{\alpha}$ of rank $r_{\alpha}$. Let $A_{t}=M_{t} \oplus W_{t}$ be obtained as a Type II deformation of $A$, with $W_{t}=\Sigma_{1} \oplus \cdots$ $\oplus \Sigma_{\sigma}$ a direct sum of total $K$-matrix algebras. Then

(a) the radical $N=M \oplus U$ where $M$ is a nilpotent ideal in $A$ and $U$ is a nilpotent subalgebra and an S-bimodule; also

(b) the index set $\{1, \ldots, s\}$ partitions into sets $\langle 1\rangle, \ldots,\langle\sigma\rangle$ with $\sigma<s$ such that the subalgebra $U \oplus S$ of $A$ is a direct sum of pre-matrix algebras $P_{p}$ with $p=1, \ldots, \sigma$, and

(c) there exist integers $n_{p q}$ with $p, q=1, \ldots, \sigma$ such that

$$
\begin{aligned}
\operatorname{dim}_{k} N_{\alpha \beta} & =r_{\alpha} r_{\beta}\left(1+n_{p p}\right), & & \text { for all distinct } \alpha, \beta \in\langle p\rangle, \\
& =\left(r_{\alpha}\right)^{2} n_{p p}, & & \text { for } \alpha=\beta \in\langle p\rangle, \\
& =r_{\alpha} r_{\beta} n_{p q}, & & \text { for } \alpha \in\langle p\rangle, \beta \in\langle q\rangle \text { and } p, q \text { distinct. }
\end{aligned}
$$

(vi) Now we may construct semirigid algebras at will. Form a semisimple $S=S_{1} \oplus \cdots \oplus S_{s}$ and then an $S$-bimodule $N$ satisfying $N_{\alpha \alpha}=(0)$ and failing one of the dimensional conditions just obtained. Define $N^{2}=(0)$. By Theorems 2 and 4, $A=N \oplus S$ is semirigid. 
(vii) We continue to suppose that $\pi_{t}$ gives a Type II deformation of $A$, as in Theorem 4. In this paragraph we construct an equivalent deformation of a very simple form,-at least where the multiplication involves elements of $W_{t}$. The simplification is accomplished in two steps: the first treats multiplication in $W_{t}$, the second the regular action of $W_{t}$ on the radical $M_{t}$.

First, since $P_{p}$ is a pre-matrix algebra, we know from [1] that there is a $k$-basis for $P_{p}^{\prime}$ (cf. the Reduction Principle of (ii) above) of the form $z_{\alpha \beta 0}$ with $\alpha, \beta \in\langle p\rangle$ (here $\left.z_{\alpha \alpha 0}=e_{\alpha}\right)$ wherein $\pi_{t}$ is equivalent to the multiplication $\Pi_{t}\left(z_{\alpha \beta 0}, z_{\beta \gamma 0}\right)=t^{m} z_{\alpha \gamma 0}$; here the $m=m_{\alpha \beta \gamma}$ are given as the solution in positive integers of the system

$$
m_{\alpha \beta \gamma}+m_{\alpha \gamma \delta}=m_{\alpha \beta \delta}+m_{\beta \gamma \delta} \text { with } m_{\alpha \beta \gamma}=0 \text { iff } z_{\alpha \beta 0} z_{\beta \gamma 0} \neq 0 .
$$

Here $\alpha, \beta, \gamma \in\langle p\rangle$ and $p=1, \ldots, \sigma$.

It follows, therefore, that there is a $K$-linear automorphism $\Phi_{t}: V_{K} \rightarrow V_{K}$ of the form $\Phi_{t}(x)=x+t \phi_{1}(x)+\cdots$ which is the identity on $M_{K}$ such that the composition $\Phi_{t} \circ \pi_{t} \circ\left(\Phi_{t}^{-1} \times \Phi_{t}^{-1}\right)$ gives a deformation of $\pi$ equivalent to $\pi_{t}$ and equals the multiplication $\Pi_{t}$ in each $P_{p}$. We agree to call this new multiplication $\pi_{t}$ also.

Now we deal with the left regular action of $W_{t}$ on $M_{t}$ determined by $\pi_{t}$. It suffices to consider the action of a matrix algebra $\Sigma_{p}$ on a $p q$-block $\left(M_{i}\right)_{p q i}$. By the Reduction Principle and Theorem 4, therefore, we need only consider the multiplications $\pi_{t}\left(z_{\alpha \beta 0}, z_{\beta \gamma i}\right)$ with $\alpha, \beta \in\langle p\rangle, \gamma \in\langle q\rangle$ and $i \geqq 1$. Just as in [1], the key observation is that such a product is a scalar (in $K$ ) multiple of a single basis element; that is, $\pi_{t}\left(z_{\alpha \beta 0}, z_{\beta \gamma i}\right)=\xi z_{\alpha \gamma t}$ with $\xi=\xi_{\alpha \beta \gamma 0 t}$ an element of $k[[t]]$. We factor $\xi=t^{m} \kappa$ where $m=m_{\alpha \beta \gamma 0 i}$ and $\kappa=\kappa_{\alpha \beta \gamma 0 i}$ is a power series in $t$ with nonzero constant term. Moreover, it is straightforward that the $z_{\beta \gamma i}$ may be chosen so that $m_{\alpha \beta \gamma 0 i}=0$ iff $\kappa_{\alpha \beta \gamma 0 i}$ has constant term 1 iff $z_{\alpha \beta 0} z_{\beta \gamma_{\circ}}=z_{\alpha \gamma_{i}}$ in $A^{\prime}$.

Now let us write $\Pi_{t}\left(z_{\alpha \beta 0}, z_{\beta \gamma}\right)=t^{m} z_{\alpha \gamma i}$ with $m=m_{\alpha \beta \gamma 0 i}$ as before; it is easy to check that this too defines a left action of the matrix algebra $\Sigma_{p}$ on $\left(M_{t}\right)_{p q i}$. Note that we have for $\alpha, \beta \in\langle p\rangle, \gamma \in\langle q\rangle$,

$$
m_{\alpha \beta \gamma 00}+m_{\alpha \gamma \delta 0 i}=m_{\alpha \beta \delta 0 i}+m_{\beta \gamma \delta 0 i} \text { with } m_{\alpha \beta \gamma 0 i}=0 \text { iff } z_{\alpha \beta 0} z_{\beta \gamma i} \neq 0 .
$$

Here $m_{\alpha \beta \gamma 00}=m_{\alpha \beta \gamma}$ of equation (4.1). Similarly, we define a right action of $\Sigma_{q}$ on $\left(M_{t}\right)_{p q i}$ by $\Pi_{t}\left(z_{\alpha \beta i}, z_{\beta \gamma 0}\right)=t^{m} z_{\alpha \gamma i}$. Now $m=m_{\alpha \beta \gamma i 0}$ and we have, for $\alpha, \beta \in\langle p\rangle$ and $\gamma \in\langle q\rangle$,

$$
m_{\alpha \beta \gamma i 0}+m_{\alpha \gamma \delta i 0}=m_{\alpha \beta \delta i 0}+m_{\beta \gamma \delta 00} \text { with } m_{\alpha \beta \gamma i 0}=0 \text { iff } z_{\alpha \beta i} z_{\beta \gamma 0} \neq 0 .
$$

The left and the right actions defined by $\Pi_{t}$ commute, so that $M_{t}$ is a two-sided $W_{t}$-module via $\Pi_{t}$, whence the equations

$$
m_{\alpha \beta \gamma 0 i}+m_{\alpha \gamma \delta i 0}=m_{\alpha \beta \delta 0 i}+m_{\beta \gamma \delta i 0} .
$$

Now since $W_{t}$ is semisimple, it follows as in Theorem 1 (cf. part (ii) of the proof) that the two-sided actions given by $\Pi_{t}$ and $\pi_{t}$ are each rigid. We show that they are equivalent in a technical sense akin to that of $[3, \mathrm{p} .65]$. The same method as in 
[1] works here. Thus, let $\tau$ be an indeterminate over $K$ and consider the deformation of the $\Pi_{t}$-action given by $\Theta_{\tau}\left(z_{\alpha \beta 0}, z_{\beta \gamma i}\right)=t^{m} \kappa(\tau) z_{\alpha \gamma i}$ with $m$ and $\kappa$ as above. By rigidity of $\Pi_{t}$, there exists a $K((\tau))$-linear automorphism $\Psi_{\tau}: M_{K((\tau))} \rightarrow M_{K((\tau))}$ of the form $\Psi_{\tau}(z)=z+\tau \psi_{1}(z)+\cdots$ such that $\Pi_{t}\left(z_{\alpha \beta 0}, z_{\beta \gamma i}\right)=\Psi_{\tau} \Theta_{\tau}\left(z_{\alpha \beta 0}, \Psi_{\tau}^{-1} z_{\beta \gamma i}\right)$. Substituting $t$ for $\tau$ shows that the actions given by $\Pi_{t}$ and $\pi_{t}$ are equivalent. Thus we have simplified $\pi_{t}$ as follows.

Theorem 5. Let $A$ and $A_{t}$ be as in Theorem 4, and let $\pi_{t}$ determine the Type II deformation. Then

(a) the linear equations $(4.1), \ldots,(4.4)$ are together solvable in positive integers, and

(b) there is a $k$-basis $z_{\alpha \beta i \lambda \mu}$ for $A$, with $\alpha, \beta=1, \ldots, s$ and $\lambda=1, \ldots, r_{\alpha}, \mu=1, \ldots$, $r_{\beta}$, and a deformation $\Pi_{t}$ equivalent to $\pi_{t}$ which has the simple form $\Pi_{t}\left(z_{\alpha \beta i \lambda \mu}, z_{\beta \gamma j \mu \nu}\right)$ $=t^{m} z_{\alpha \gamma h \lambda v}$ whenever one of $i, j$ is zero. Here $h=\max (i, j)$ and $m=m_{\alpha \beta \gamma i j}$ is given by the solution of the equations in (a).

Thus the deformation of those multiplications involving the subalgebra $U \oplus S$ of $A$ may always be expressed in terms of polynomials, rather than power series, in $t$. Also, if the radical is small in the sense that all $n_{p q} \leqq 1$ (cf. Theorem 4), then we claim that the factorization trick may be used in $M$, so that Type II deformability implies deformability in terms of polynomials in $t$; there is a $\Pi_{t}^{\prime}=\pi+t F_{1}+\cdots$ $+t^{n} F_{n}$ for some $n$.

(vii) We have now amassed enough information about Type II deformability to state a sufficiency theorem. This will reduce the problem of deforming $A$ to that of constructing a deformation of a nilpotent ideal $M$, subject to certain constraints. To see this, we make a further observation about $\Pi_{t}$ above. Denote by $\mu_{t}$ the restriction of $\Pi_{t}$ to the ideal $M$ of Theorem 4 . Also, write $\Pi_{t}(x, y)=x * y$ for brevity. Now let $u \in U \oplus S$ and $z^{\prime}, z^{\prime \prime} \in M$. Then the associativity of the products $u * z^{\prime} * z^{\prime \prime}$, $z^{\prime} * u * z^{\prime \prime}, z^{\prime} * z^{\prime \prime} * u$ imposes constraints on $\mu_{t}$, considered as a deformation of $M$. These constraints could be written as explicit formulae involving factors $t^{m}$ and the structure constants for $\mu_{t}$, but we refrain from doing this. We shall say in this case that $\mu_{t}$ associates with $\Pi_{t}$ (with $\Pi_{t}$ here considered as a deformation of the action of $U \oplus S$ on $M$ ).

The following definition isolates the structural properties of Theorem 4 and will simplify the statement of the theorem. Let $A=N \oplus S$ and $N_{\alpha \beta}$ be as above. A partition $\langle 1\rangle,\langle 2\rangle, \ldots,\langle\sigma\rangle$ of the index set $\{1, \ldots, s\}$ with $\sigma<s$, is said to determine a Type II structure on $N$ iff each $N_{\alpha \beta}$ decomposes into $\alpha \beta$-blocks $N_{\alpha \beta i}$, with $i=0,1,2, \ldots$ when $\alpha, \beta$ are distinct elements of some set $\langle p\rangle$ and $i=1,2, \ldots$ otherwise, such that (1) $N_{\alpha \beta 0} N_{\beta \gamma i} \subset N_{\alpha \gamma i}$ and $N_{\gamma \beta i} N_{\beta \alpha 0} \subset N_{\gamma \alpha i}$ for all distinct $\alpha, \beta \in$ $\langle p\rangle$ and all $\gamma$, and (2) the dimension of $N_{\alpha \beta}$ satisfies (c) of Theorem 4. Given a Type II structure on $N$, there is a $k$-basis $z_{\alpha \alpha 0}\left(=e_{\alpha}\right)$ for $S^{\prime}, z_{\alpha \beta i}$ for $N^{\prime}$ with $i$ as above for which the equations $(4.1), \ldots,(4.4)$ have an interpretation (that is, $m_{\alpha \beta \gamma}=0$ if $z_{\alpha \beta 0} z_{\beta \gamma 0} \neq 0$, otherwise $m_{\alpha \beta \gamma}$ is a positive integer to be determined). 
Theorem 6. Let $A=N \oplus S$ over $k$ as in Theorem 4. Suppose

(a) some partititon $\langle 1\rangle,\langle 2\rangle, \ldots,\langle\sigma\rangle$ of $\{1, \ldots, s\}$ with $\sigma<s$ determines a Type II structure on $N$, and

(b) the equations (4.1), .., (4.4) have a solution in positive integers.

Then define $\Pi_{t}\left(z_{\alpha \beta i}, z_{\beta \gamma j}\right)=t^{m} z_{\alpha \gamma h}$ whenever one of $i, j$ is zero, where $h=\max (i, j)$ and $m=m_{\alpha \beta \gamma i j}$ is given by the equations above. Suppose further

(c) there exists a nilpotent deformation $\mu_{t}$ of $M=\oplus N_{\alpha \beta i}$ with $i \geqq 1$ which associates with $\Pi_{t}$.

Then $A$ admits a Type II deformation.

This theorem can be used to answer the question: "which $k$-algebras $A$ deform into the semisimple algebra $\Sigma_{1} \oplus \cdots \oplus \Sigma_{\sigma}$, where each $\Sigma_{p}$ is a total $K$-matrix algebra?" In this case the general problem of constructing a deformation (which involves solving for an infinite number of 2-cochains $F_{1}, F_{2}, \ldots$ in sequence) reduces to finding integer solutions for a single finite overdetermined system of linear equations.

\section{BIBLIOGRAPHY}

1. F. J. Flanigan, Which algebras deform into a total matrix algebra?, Bull. Amer. Math. Soc. (to appear).

2. - Algebraic geography: varieties of structure constants, Pacific J. Math. (to appear).

3. M. Gerstenhaber, On the deformation of rings and algebras, Ann. of Math. 79 (1964), 59-103.

4. - On the construction of division rings by the deformation of fields, Proc. Nat. Acad. Sci. U.S.A. 126 (1966), 690-692.

5. A. Nijenhuis and R. Richardson, Cohomology and deformations in graded Lie algebras, Bull. Amer. Math. Soc. 72 (1966), 1-29.

6. S. Page and R. Richardson, Stable subalgebras of Lie algebras and associative algebras, Trans. Amer. Math. Soc. 127 (1967), 302-312.

7. B. L. van der Waerden, Modern algebra, Vol. II, Ungar, New York, 1950.

University of Pennsylvania,

Philadelphia, Pennsylvania 